\title{
Design considerations of high RAP-content asphalt produced at reduced temperatures
}

\author{
Ahmed Abed $\cdot$ Nick Thom $\cdot$ Davide Lo Presti $\mathbb{B}$
}

Received: 1 January 2018/Accepted: 28 June 2018/Published online: 3 July 2018

(C) The Author(s) 2018

\begin{abstract}
In many countries recycling of reclaimed asphalt pavement (RAP) for road surface layers is limited to a maximum of $10-30 \%$. This is due to technical limitation of common asphalt plant but also to specifications that are still restrictive when it comes to increasing RAP in surface courses. The mistrust in this practice is mainly related to uncertainty in performance of these mixes as well as to existing fundamental issues with the mix design, especially when production temperatures are lowered. This paper analyses some of the factors affecting the design of warm asphalt mixtures for surface course layers containing 50\% RAP, and suggests a framework to justify the common assumption of full blending by optimising production conditions. A control hot mix asphalt which was manufactured with $49 \mathrm{dmm}$ penetration binder and asphalt mixtures containing 50\% RAP produced at temperatures between 95 and $135^{\circ} \mathrm{C}$ and at different mixing times were investigated in terms of volumetric properties, indirect tensile stiffness modulus, and indirect tensile strength. The highcontent RAP mixtures were produced within the warm mix region by using only a very soft binder as a rejuvenator, which reduces production costs. Statistical analysis was deployed, and different models were
\end{abstract}

A. Abed $\cdot$ N. Thom $\cdot$ D. Lo Presti $(\square)$

Nottingham Transportation Engineering Centre, Faculty of Engineering, The University of Nottingham, University Park, Nottingham NG7 2RD, UK

e-mail: davide.lopresti@nottingham.ac.uk developed to estimate degree of blending between RAP binder and rejuvenator binder, and to predict the equivalent penetration of the blend without binder extraction and recovery. The analysis results showed that the selected performance indicators correlate significantly with mixing time and temperature, and provide evidence that only in certain circumstances and if the production conditions are accurately controlled, the practical full blending approach is acceptable.

Keywords Reclaimed asphalt pavement (RAP) . Degree of blending (DoB) · Full blending · Mix design - Warm mix asphalt

\section{Introduction}

Warm mix asphalt (WMA) can be defined as an asphaltic mixture produced at $10-40{ }^{\circ} \mathrm{C}$ lower than traditional HMA [1]. This mixture has several economic and environmental benefits such as reduced fuel consumption and less greenhouse gas emissions during the asphalt production phase [2, 3]. Based on these advantages and the increased awareness of economic and environmental issues, the WMA industry has gained global acceptance with increased demand between 2009 and 2014 [4]. On the other hand, RAP is now considered as a valuable material 
and a satisfactory replacement for virgin aggregate and binder due to the economic and environmental benefits associated with the recycling process [5]. RAP recycling has become a familiar practice since the 1970s due to inflation of crude oil prices which increased asphalt pavement material costs [6]. RAP recycling at WMA production temperatures would, therefore, be a promising approach to further enhance the sustainability of the asphalt industry. Giani et al. [7] stated that asphalt recycling with WMA technology is the best approach to achieving a sustainable and environmentally friendly asphalt industry. For this reason, many highway agencies nowadays are investigating the applicability and feasibility of these kinds of asphaltic mixture.

Until 2006, RAP incorporation in the US had been limited to around $15 \%$ because no binder grade change was required at this level and no further binder testing was required. Nevertheless, the sharp increase in bitumen prices in that year significantly increased the demand to produce asphalt mixtures with higher RAP contents [5]. More recently, RAP recycling in asphaltic mixtures has usually been limited to $30 \%$ [8], with $20 \%$ average RAP recycling in the United States in 2014 [4]. This may be related to several factors such as RAP agglomeration, poor blending of RAP and virgin binder, variability in RAP binder characteristics, and even government legislation [9]. Technical factors have the potential to significantly affect the homogeneity and performance of mixtures containing RAP (AC-RAP).

In this regard, several researchers have investigated the interaction and blending between RAP and virgin asphalt materials, and different methods have been proposed to simulate and understand these phenomena. Shirodkar et al. [10] defined the DoB as the percentage of RAP binder that will be active in the mix. They suggested an iterative procedure to calculate the DoB based on the superpave critical hightemperature parameter $G^{*} / \sin \delta$ for binders recovered from predefined sizes of RAP and virgin aggregate particles. Their method was to assume an initial percentage of RAP binder that will be active in the mix, then design and manufacture samples based on this assumption, after that to sieve and isolate RAP from aggregate particles, and extract and recover binder from both portions to run DSR testing. The DoB was then determined using the following equation:

$$
\begin{aligned}
& \text { Degree of partial blending (\%) } \\
& =100 \times\left|1-\frac{\left|\mathrm{CHTP}_{\mathrm{vab}}-\mathrm{CHTP}_{\mathrm{rab}}\right|}{\left|\mathrm{CHTP}_{\mathrm{vb}}-\mathrm{CHTP}_{\mathrm{rb}}\right|}\right|
\end{aligned}
$$

where CHTP is the critical high-temperature parameter $\left(G^{*} / \sin \delta\right)$ for the designated binder, vab is the binder recovered from the virgin aggregate, rab is the binder recovered from RAP aggregate, vb is the virgin binder, and rb is the RAP binder. If the calculated DoB is close to the assumed percentage of RAP binder participation then the correct DoB has been obtained; if not, then a new value for RAP binder participation has to be assumed, and DoB has to be calculated again until these two values converge. They reported 70 and 96\% DoB for 25 and 35\% RAP incorporation respectively. However, the DoB can be affected by the production conditions for the same RAP incorporation level and properties, because increasing mixing temperatures reduces RAP binder viscosity which leads to higher RAP binder activation. Coffey et al. [11] defined the DoB as the mobilisation percentage of RAP binder in an asphalt mix. They estimated the DoB based on air voids content by assuming the initial DoB is $70 \%$ based on literature, if this assumption was true then the air voids at 75 gyrations has to be $4 \%$, if not then the binder content has to be adjusted based on a revised DoB percentage. They reported a DoB of $84.7-90 \%$ for three different RAP sources with $25 \%$ RAP incorporation. However, this level of RAP incorporation was relatively low.

Bowers et al. [12] studied blending and diffusion between the RAP binder and virgin bitumen using staged extraction to isolate the recovered binder into different layers. Gel Permeation Chromatography and Fourier Transform Infrared Spectroscopy tests were used to study the properties of the binder recovered from different layers, and it was concluded that blending occurs in all binder layers but that it may not be uniform. Bressi et al. [13] applied the same concept of isolating RAP particles from virgin aggregate particles to analyse the recovered binder from both aggregate portions, and to quantify RAP clustering. They found that at high RAP contents, clustering is reduced due to the lower amount of virgin aggregate which plays an essential role in activating and removing the aged binder from RAP particles by mechanical abrasion. However, this could also mean that less RAP binder will be activated and ultimately that a limited RAP content can be incorporated. 
Another study conducted by Hassan et al. [14] to understand the effect of mixing time on the homogeneity of AC-RAP mixtures by means of computed tomography and optical microscopy concluded that doubling the mixing time improved the distribution of the air voids within the specimens produced, which shows the importance of this factor.

\subsection{Aim of the study}

Most of the above mentioned studies depend on the idea of separating RAP particles from virgin aggregate particles to recover and study the binder properties from both portions. However, analysing the characteristics of the blended binders and predicting the resultant binder grade without binder extraction and recovery is still a challenge. Furthermore, assuming that DoB less than $100 \%$ simply means that part of the RAP binder is inactive, it is unclear which aggregate gradation should be assumed, the gradation after fully extracting the binder or the gradation when the RAP aggregate is coated with an unknown thickness of inactive RAP binder, nor how to determine this gradation. It is also unclear wether the inactive binder will have any effect on the mixture's long-term performance. Because the hard binder will be in contact with a lower viscosity binder for a long time, some diffusion between the two binders might occur, and that could change the properties of the mix.

These points are quite critical because the volumetric properties will be incorrectly designed and eventually performance will be affected. Accordingly, the authors believe that a good approach to addressing this problem is to keep the common assumption that RAP binder is completely mobilised (full blending) and to design the mix based on RAP aggregate gradation. Then the production conditions and the mix design have to be revised (i.e., increasing mixing temperature, using additives, reducing RAP content, increasing mixing time, etc.) to achieve this assumption. In this case, the mix gradation will match the designed aggregate gradation, and the RAP binder will be thoroughly blended with the soft binder. Moreover, the performance of AC-RAP mixtures has to be evaluated to understand and validate the applicability of incorporating RAP. Therefore, this paper suggests a framework to predict the DoB between RAP and virgin materials, and to optimise production conditions to achieve full blending by a simple but effective and practical procedure. Furthermore, to investigate and analyse the effects of two of the most critical parameters namely mixing time and temperature on mixture homogeneity and DoB level, three indicators, namely volumetric properties (air voids), indirect tensile stiffness modulus (ITSM), and indirect tensile strength (ITS) were selected to quantify the effects of the chosen parameters and to estimate the DoB between RAP and virgin binders.

\section{Materials and methods}

\subsection{Materials}

To achieve the aims of this study, a 0/14 AC for surface courses according to BSEN 13108-1:2006 [15] was selected as a reference mix. Two mixtures were designed, one without any RAP (the control HMA), and one matching the control HMA with respect to aggregate gradation, binder content and volumetric properties, but containing 50\% RAP as a percentage of aggregate content. The following materials were used in the experimental work:

1. $40 / 60$ binder grade with $49 \mathrm{dmm}$ penetration, used for the control HMA.

2. $160 / 220$ binder grade with $188 \mathrm{dmm}$ penetration, used as a rejuvenator. This binder was selected based on blending charts as will be explained in the following sections.

3. Granite aggregate supplied from Bardon Hill quarry, Leicestershire, UK. The aggregate consisted of four fractions; 0-4, 4-6, 6-10, and $10-14 \mathrm{~mm}$, so by mixing a certain percentage of each size, the final aggregate grading met the required 0/14 AC aggregate gradation, as shown in Fig. 1.

4. RAP supplied by FM Conway, London, UK, obtained by milling of road surfaces. The compositional analysis of the RAP revealed that the RAP contained $5.7 \%$ binder with a penetration 17 dmm. It also showed that the RAP aggregate consisted of fractions as shown in Fig. 1. So by analysing RAP aggregate grading and mixing $50 \%$ of the RAP aggregate with specific proportions of the virgin aggregate, the final grading of AC-50\% RAP almost matched the control HMA grading as shown in Fig. 1. 


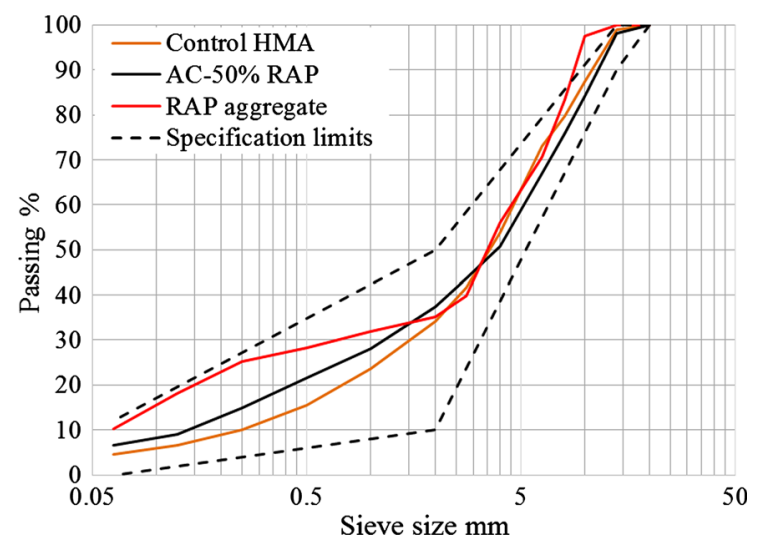

Fig. 1 Aggregate gradation of the control HMA aggregate, RAP aggregate only, and AC mix containing 50\% RAP and required virgin aggregate

It has to be mentioned that every effort has to be made to bring the gradation of the mix containing RAP as close as possible to the control mix, to eliminate or minimise any effects of differences in the aggregate gradation on the performance of the studied mixtures. Also, no WMA technology was implemented in this study, because the viscosity of the soft binder that was used in this study was sufficiently low for it to be mixed without using any WMA additives.

\subsection{Mix design}

The control HMA was designed according to British Standards. As stated earlier, 0/14 mm AC surface course was selected in this study. Binder content was determined as 5.1\% according to BS 4987-1 [16]. The maximum theoretical specific gravity was determined according to BS EN 12697-5 [17] by following the mathematical procedure using the following equation:

$\mathrm{Dmc}=100 /\left(p_{\mathrm{b}} / d_{\mathrm{b}}+p_{\mathrm{a} 1} / d_{\mathrm{a} 1}+p_{\mathrm{a} 2} / d_{\mathrm{a} 2}+\cdots+p_{\mathrm{an}} / d_{\mathrm{an}}\right)$

where Dmc is the maximum density by calculation, $P_{\mathrm{b}}$ and $d_{\mathrm{b}}$ are percentage and density of the binder and $p_{\mathrm{ai}}$ and $d_{\mathrm{ai}}$ are percentages and densities of every aggregate portion. Mixing and compaction temperatures were determined based on dynamic viscosity measurements. Bitumen viscosities of 0.17 and $0.28 \mathrm{~Pa} \mathrm{~s}$ were recommended for mixing and compaction [18]. Accordingly, the mixing and compaction temperatures were determined as 155 and $145^{\circ} \mathrm{C}$.
Asphalt mix design with RAP incorporation is a complicated process. Due to the variability in RAP characteristics from one source to another or even from the same source, there is no globally applicable mix design method. Lo Presti et al. [9] stated that due to the variability in RAP properties, every RAP should be characterised accurately with particular attention paid to the RAP binder. In general, there are two ways to design RAP mixtures. The first is to design based on a known RAP percentage; the second is to design based on a known virgin binder grade [8]. The former is useful in controlling the RAP quantity in the mix design, but the required virgin binder grade has to be determined based on blending charts. The latter is useful when the grade of the virgin binder that will be mixed with the RAP is known, based on which the RAP quantity can be determined. In this study, since the chosen RAP quantity was $50 \%$, the mix was designed based on the known RAP quantity approach.

The detailed design procedure followed in this study was as follows:

1. Based on the nominal aggregate size of the control mix, the RAP was sieved on sieve size $16 \mathrm{~mm}$ and all particles larger than this size were removed.

2. Rap binder extraction and recovery was carried out according to BS EN 12697-3:2013 [19]. The extraction and recovery process is required for design purposes only.

3. Determinate RAP binder content according to BS EN 12697-1:2012 [20].

4. Measure the particle size distribution of RAP aggregate after extracting the binder according to BS EN 12697-2:2015 [21].

5. Determinate RAP maximum theoretical specific gravity according to British Standard BS EN 12697-5 following the volumetric procedure.

6. Determinate the RAP aggregate effective specific gravity using the following equation [22]:

$$
\begin{aligned}
\text { Gse }_{\text {RAP aggregate }}= & \left(100-\mathrm{RAP}_{\mathrm{bc}}\right) /\left(100 / \mathrm{Gmm}_{\mathrm{RAP}}\right. \\
& \left.-\mathrm{RAP}_{\mathrm{bc}} / \mathrm{Gb}_{\mathrm{RAP}}\right)
\end{aligned}
$$

where Gse $_{\mathrm{RAP} \text { aggregate }}$ is the effective specific gravity of the RAP aggregate, $\mathrm{Gmm}_{\mathrm{RAP}}$ is the maximum theoretical specific gravity of the RAP, $\mathrm{RAP}_{\mathrm{bc}}$ and $\mathrm{Gb}_{\mathrm{RAP}}$ are RAP binder content and the specific gravity of the RAP binder. Gse of the 
RAP aggregate was determined to calculate the maximum theoretical specific gravity of the ACRAP mix using Eq. 1, so that it can be used to calculate air voids content in the samples.

7. Calculate the required mass of RAP aggregate based on designed RAP\% as follows:

$\mathrm{RAP}_{\text {agg }}=\mathrm{RAP} \% \times$ total aggregate mass

where $\mathrm{RAP}_{\text {agg }}$ is the mass of RAP aggregate, $\mathrm{RAP} \%$ is the designed percentage of the RAP in the mix.

8. Calculate the mass of RAP binder that will be introduced into the mix, as follows:

$\operatorname{RAP}_{\mathrm{b}}=\operatorname{RAP}_{\mathrm{agg}} \times\left(\frac{1}{1-\mathrm{RAP}_{\mathrm{bc}}}-1\right)$

where $\mathrm{RAP}_{\mathrm{b}}$ is the mass of RAP binder. Accordingly, the RAP mass that has to be introduced into the mix is basically the sum of RAP aggregate weight calculated using Eq. 4 and RAP binder weight using Eq. 5.

9. Calculate the mass of the rejuvenator binder and the percentage of each binder with respect to the total binder weight in the mix, as follows:

$\operatorname{Rej}_{\mathrm{b}}=$ Total binder mass $-\mathrm{RAP}_{\mathrm{b}}$

$\operatorname{Rej}_{\mathrm{b} \%}=\operatorname{Rej}_{\mathrm{b}} /$ Total binder mass

$\mathrm{RAP}_{\mathrm{b} \%}=\mathrm{RAP}_{\mathrm{b}} /$ Total binder mass

where $\mathrm{Rej}_{\mathrm{b}}$ is the mass of the required soft binder in the mix, $\operatorname{Rej}_{b \%}$ is the percentage of the soft binder and $\mathrm{RAP}_{\mathrm{b} \%}$ is the percentage of the RAP binder with respect to the total binder weight in the mix.

10. After determining the percentages of each of the binders, the required grade of the rejuvenator can be calculated using the following logarithmic equation [15]

$$
\begin{aligned}
& \log \text { pen }_{\text {blend }}= a \times \log \text { pen }_{\text {RAP binder }}+b \\
& \times \log \text { pen } \\
& \text { rejuvenator }
\end{aligned}
$$

Doing the required mathematical manipulation, the grade of the rejuvenator binder that brings the RAP binder into a specific binder grade can then be calculated as follows: pen $_{\text {rejuvenator }}=10^{\left(\left(\log \text { pen }_{\text {blend }}-a \times \log \text { pen }_{\mathrm{RAP} \text { binder }}\right) / b\right)}$

where pen $_{\text {rejuvenator }}$ is the required binder grade, pen $_{\text {blend }}$ is the target grade which is the grade of the control mix binder, pen RAP $_{\text {is }}$ the grade of the RAP binder, $a$ and $b$ are the proportions of RAP binder and rejuvenator respectively.

\subsection{Blending charts and blended binder properties}

Based on the suggested mix design procedure, the proportions of the rejuvenator and RAP binder were determined, while the rejuvenator grade was calculated based on Eq. 9. For 50\% RAP aggregate incorporation, RAP binder percentage was $44 \%$ of the total binder. With these conditions and using a RAP binder of penetration $17.3 \mathrm{dmm}$ and setting the target binder to $49.0 \mathrm{dmm}$, the soft binder grade required was $188 \mathrm{dmm}$. Figure 2 shows the proposed blending chart for the blended binders, and Table 1 shows penetration test results of the blended binders based on the calculated proportions. It can be seen that the rejuvenated binder penetration $(47.3 \mathrm{dmm})$ is quite close to the target binder penetration $(49.0 \mathrm{dmm})$ which implies successful RAP binder rejuvenation and the use of the correct binder proportions. Moreover, Fig. 3 presents the results of rotational viscosity tests of all studied binders conducted using a Brookfield viscometer. The results indicate that blending of the RAP binder with the soft binder based on the suggested proportions results in a rejuvenated binder with quite comparable viscosity to the target binder which confirms the previous conclusion. Furthermore, to further validate the results of the suggested blending proportions, rheological properties of all binders were tested using a DSR by running frequency sweep tests and constructing complex modulus master curves. Figure 4 shows the constructed shear modulus master curves. The effect of the soft binder on the rheological properties of the hard binder can clearly be seen, also the match between the rejuvenated binder and the target binder properties, which suggests the recovery of the RAP binder's rheological properties. Moreover, Fig. 5 is an isochronal plot of the parameter $G^{*} / \sin \delta$ at $10 \mathrm{rad} / \mathrm{sec}$ frequency. The rejuvenation process using the soft binder shifted the $G^{*} / \sin \delta$ results to values 
Fig. 2 RAP and soft binder blending chart

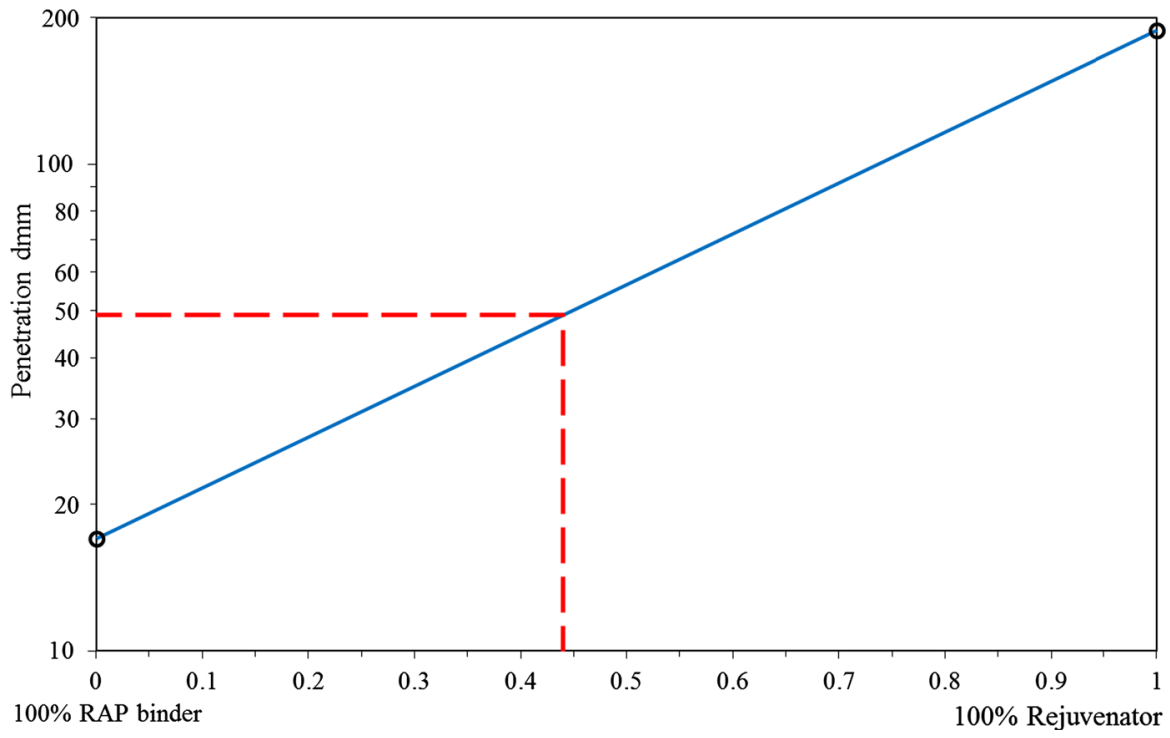

Table 1 Properties of the RAP binder, rejuvenator, and the base binder

\begin{tabular}{llccc}
\hline Binder & RAP binder & Rejuvantor & Base binder & Rejuvenated binder \\
\hline Penetration $(\mathrm{dmm})$ & 17.3 & 188.3 & 49.0 & 47.7 \\
Critical high temperature $\left({ }^{\circ} \mathrm{C}\right)$ & 81.6 & 57.8 & 69.8 & 71.2 \\
\hline
\end{tabular}

Fig. 3 Rotational viscosity results

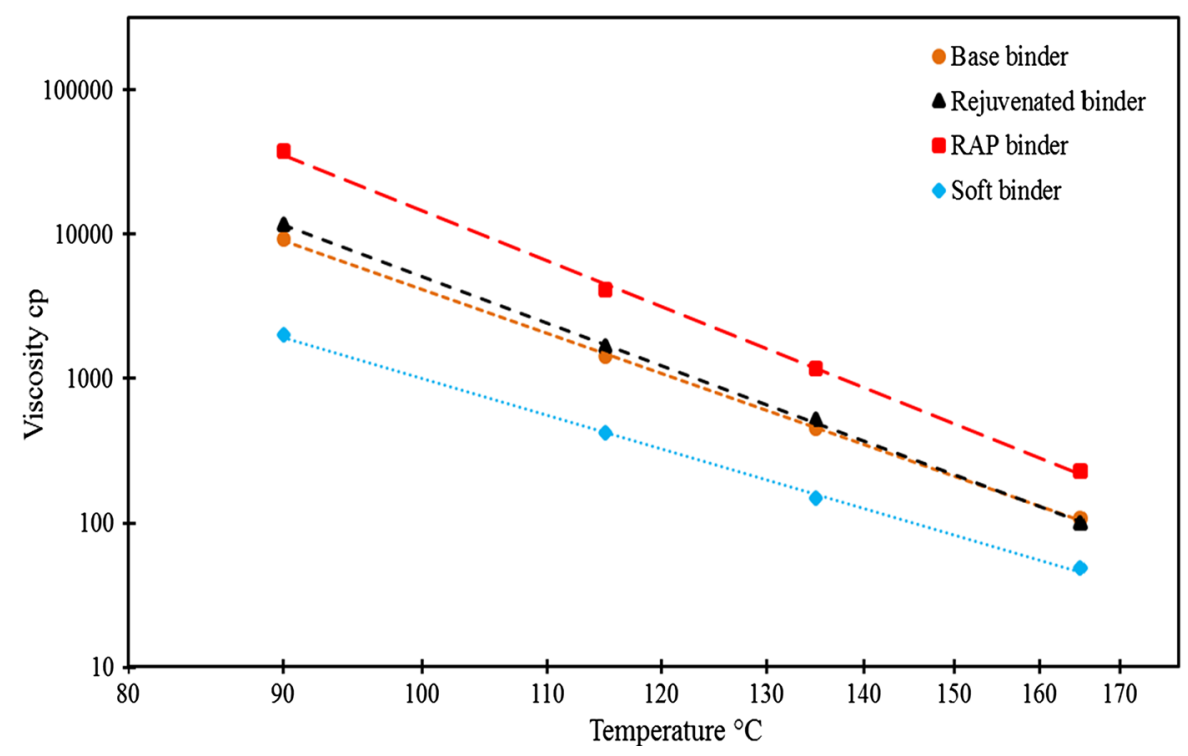

close to those of the target binder, which means that the rejuvenated binder has similar rheological properties to the target binder. Also, the critical high temperature which is the temperature at which the parameter $G^{*} / \sin \delta$ equals $1.0 \mathrm{kPa}$ [23] was determined as shown in Table 1. The results show that the critical high temperatures for the target binder and the rejuvenated binder are quite similar, with a difference of $1.4{ }^{\circ} \mathrm{C}$ which means that both binders have a similar critical high temperature. 
Fig. 4 Complex modulus master curves

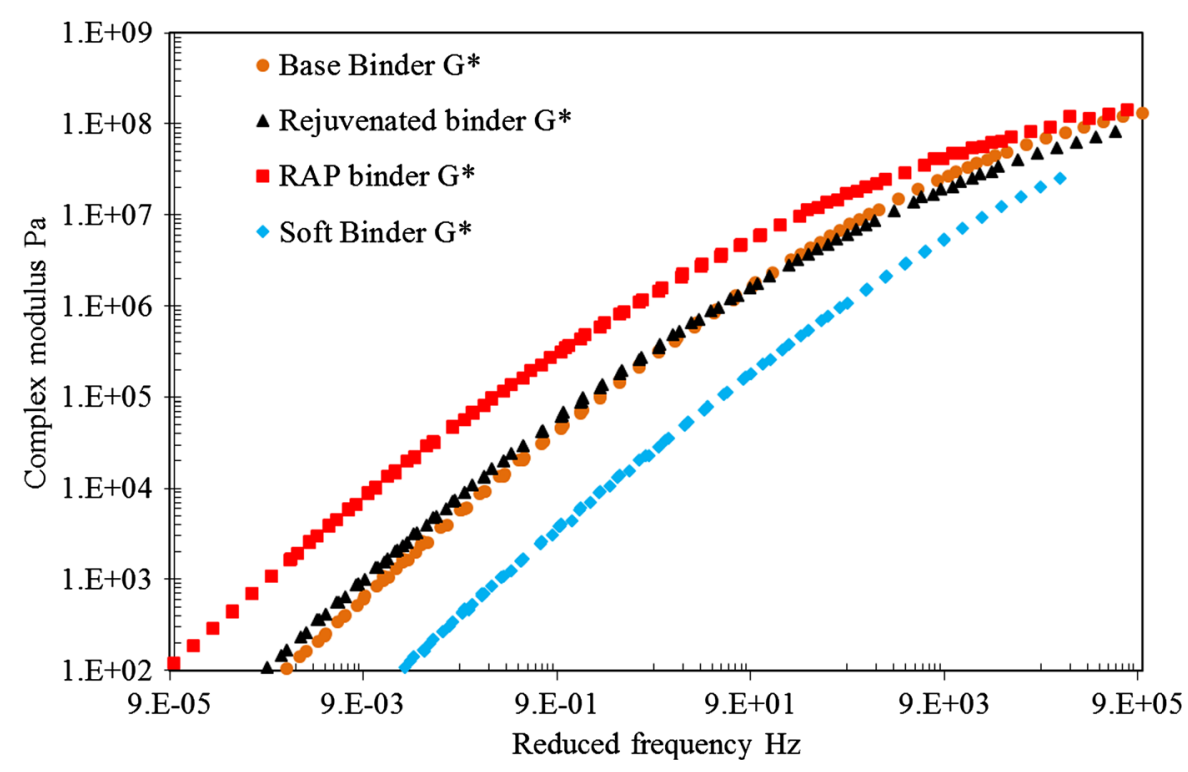

Fig. 5 Critical hightemperature parameters

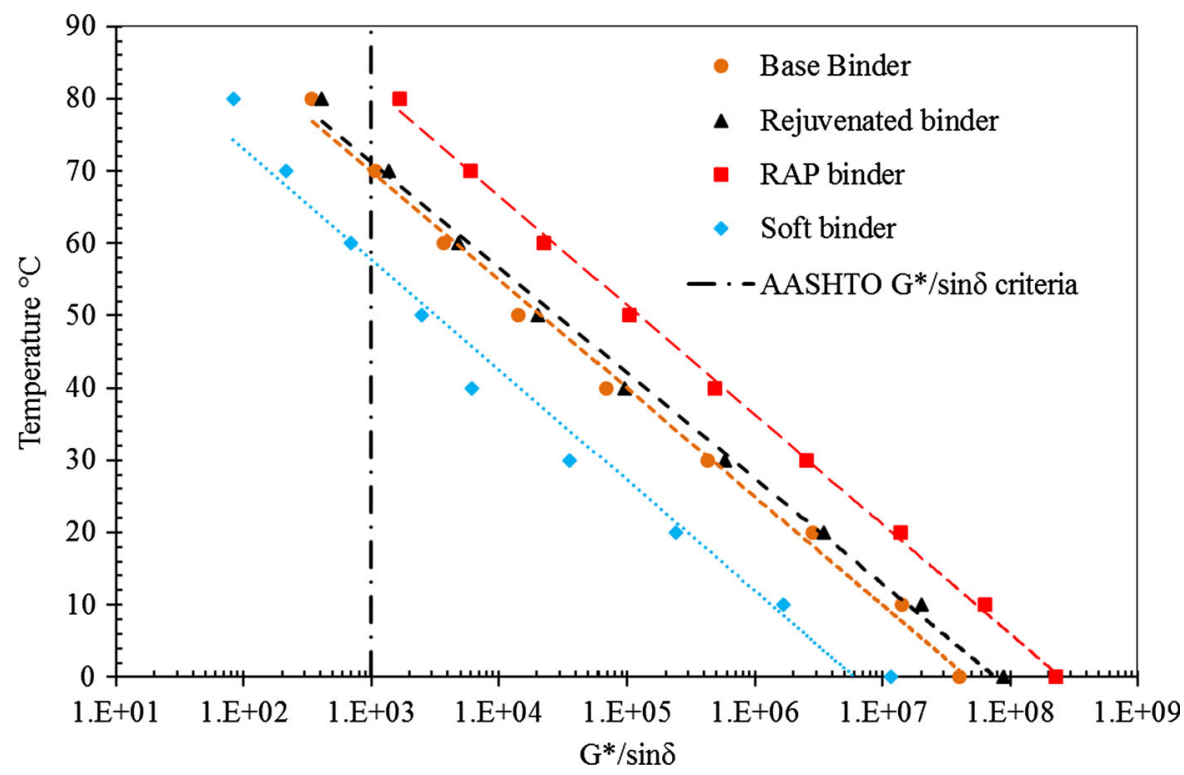

These results suggest that Eq. 9 has accurately determined the grade of the required soft binder, and binder blending based on the penetration criterion can satisfy the superpave performance grade requirements since both binders have a similar critical high temperature after the rejuvenation process. The results also suggest that both binders will perform similarly if the blending between the soft binder and RAP binder is $100 \%$. This point is quite crucial because estimating
RAP binder participation and the degree of blending depends on this assumption; if the two binders are $100 \%$ blended in the mix, then a similar performance to that of the control HMA should be obtained. If the blending is less than $100 \%$, a difference in the performance between RAP-AC and HMA will be observed, which also means that the production conditions have to be adjusted until similar performance to the control HMA is obtained. 


\subsection{Asphalt mixing and compaction}

After determining the mix design proportions, the control HMA was mixed and compacted according to the standard production temperatures of $40 / 60$ binder grade. The aggregate was heated overnight, and the binder was heated for $3 \mathrm{~h}$; then the ingredients were mixed in a bucket mixer and compacted at the designated compaction temperature in square moulds to prepare slabs with dimensions $306 \times 306 \mathrm{~mm}$. After that, cylindrical specimens with $100 \mathrm{~mm}$ were cored, and the top and bottom were trimmed in order to obtain homogeneous samples for testing. The choice of sample diameter was due to compliance with the testing standards used in this study to evaluate the mechanical properties of the studied mixtures. In the case of fabricating RAP samples, the following procedure was followed:

1. Heat the virgin aggregate overnight at the required mixing temperature.

2. Heat the RAP and soft binder for $2-3 \mathrm{~h}$ at the production temperature.

3. Mix the RAP and virgin aggregate only for $30 \mathrm{~s}$. This step is essential to reduce the RAP binder film and to help in preventing RAP agglomeration.

4. Add the soft binder and mix for the specified mixing time.

5. Compact the asphalt at the required compaction temperature, then core and trim normally.

Following this procedure, HMA control specimens were mixed at $155{ }^{\circ} \mathrm{C}$ and compacted at $145{ }^{\circ} \mathrm{C}$ since these temperatures correspond to the standard mixing (0.17 Pa s) and compacting viscosities ( $0.28 \mathrm{~Pa} \mathrm{~s})$ of the base binder [18]; whereas AC-RAP samples were mixed at three different temperatures within the WMA production range, 135,115 , and $95{ }^{\circ} \mathrm{C}$, and compacted at $10{ }^{\circ} \mathrm{C}$ less than the mixing temperature. At every mixing temperature, 1, 3 and 5 min mixing time were used. This choice of mixing times and temperatures was made in order to understand the effect of these factors on the blending process, giving information that can be utilised in optimizing the production conditions of AC-RAP mix. Figure 6 shows four ACRAP samples fabricated in this way but under different production conditions.
2.5 Volumetric properties and performance measures

\subsubsection{Air voids}

Figure 6 which presents four samples mixed at different temperatures for different times, demonstrates visually that the homogeneity of the samples is significantly affected by the production conditions. Figure $6 a$, b clearly show the agglomeration of RAP particles due to the lower production temperature and mixing time, whereas Fig. 6c, d show a well mixed sample. Moreover, the air voids content and distribution are significantly affected by these factors. Thus, the air voids content was used to assess the volumetric properties. It was calculated by measuring the bulk density following British Standard BS EN 12697 [24] and determined as the difference between the maximum density and the bulk density divided by the maximum density.

\subsubsection{Indirect tensile stiffness modulus (ITSM)}

This measure was selected to evaluate specifically the performance of the asphalt samples containing RAP. In this test, 5 microns horizontal deformation is applied by exerting the required stress, which equates to 50 microstrain for $100 \mathrm{~mm}$ diameter samples. Since this is a small strain, it is mainly absorbed and resisted by the binder in the mix, which plays a significant role in preventing the movement of aggregate particles [25]. In other words, this test is sensitive to binder stiffness because it is the parameter that resists the applied deformation during testing. Accordingly, it was implemented to study the effects of production conditions on the homogeneity and performance of AC-RAP mix. In this study, the stiffness was measured by performing indirect tensile stiffness modulus (ITSM) tests according to British Standard BS EN12697-26 [26].

\subsubsection{Indirect tensile strength (ITS)}

Strength is a fundamental property that can be used to evaluate the stress that a material can resist before a fracture occurs. This parameter was selected since it was reported that it correlates well with rutting and fatigue cracking; the higher the strength, the higher the rutting and fatigue cracking resistance [27]. Moreover, 

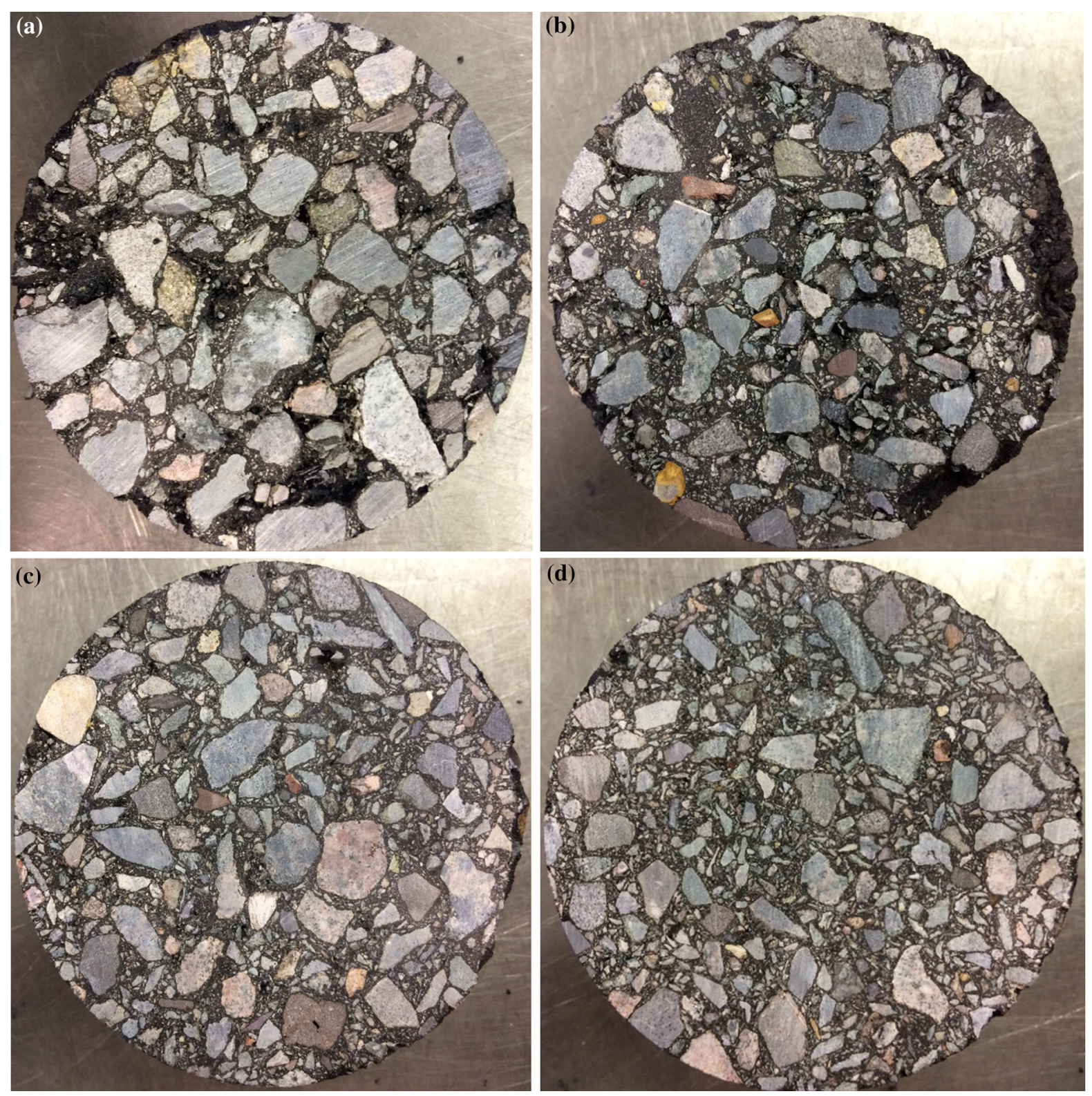

Fig. 6 a, b are AC-RAP samples containing 50\% RAP mixed at $95{ }^{\circ} \mathrm{C}$ for $1 \mathrm{~min}, \mathbf{b}, \mathbf{c}$ are also AC-RAP samples containing $50 \%$ RAP but mixed at $135{ }^{\circ} \mathrm{C}$ for $3 \mathrm{~min}$

indirect tensile strength (ITS) tests can be conducted after ITSM testing because the latter is a nondestructive test, which means the same samples can be tested. The ITS test was performed in accordance with British Standard BS EN 12697-23 [28].

\section{Results and discussion}

\subsection{Air voids}

Figure 7 presents the results of the air voids content. The results show a clear relationship between air voids content and mixing time and temperature parameters. The higher the mixing temperature, the lower the air 
Fig. 7 Air voids results

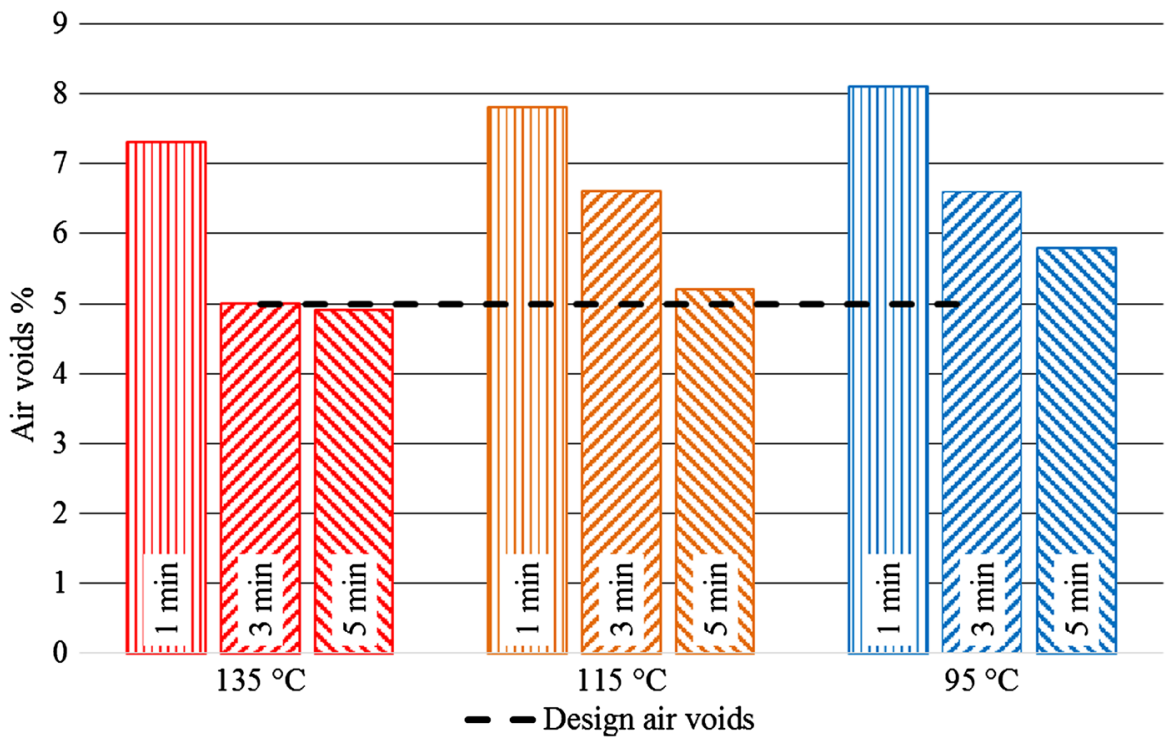

voids until it reaches the target value of 5\%. The trend is complicated by mixing time. For example, mixing at $135{ }^{\circ} \mathrm{C}$ for 3 min was sufficient to achieve the target air voids, whereas at $115^{\circ} \mathrm{C}$, a longer mixing time was required, and the target air voids were barely obtained after 5 min of mixing. At $95{ }^{\circ} \mathrm{C}$, the target air voids were never achieved, even after 5 min of mixing. The different air voids under different production conditions may be explained by two factors.

The first is the phenomenon of particle clustering. Clustering can be defined as the agglomeration of RAP particles during mixing which leads to changing effective aggregate gradation and a decrease in the volume of free fine particles in the mix, thus affecting air voids content [29]. Increasing mixing temperature reduces RAP particle clustering by lowering RAP binder viscosity during mixing. The second factor is the actual size range of RAP aggregate particles and the amount of activated binder in the mix. At low production temperatures, less RAP binder is activated and the remainder of the RAP binder is inactive, increasing the effective sizes of RAP particles. This changes the effective particle gradation from the design gradation, and leads to a dry mix since the active binder content is less than the design binder content. As the production temperature increases, the viscosity of RAP binder decreases, and more active binder is introduced into the mix, filling the air voids, and reducing the effective sizes of RAP aggregate particles toward the design gradation. This leads to lower air voids in the mix and improved volumetric properties. These explanations are consistent with the air voids results in Fig. 7.

To validate the statistical significance of these results, a $T$ test was performed, and a total number of 45 specimens (5 samples for every condition) were tested. Air void results for every parameter were verified against the air void results for the samples produced at $135{ }^{\circ} \mathrm{C}$ and 5 min of mixing, since target air voids were achieved under these conditions. Table 2 presents the results of the analysis. It can be seen that most of the results are statistically

Table 2 Air voids statistical analysis

\begin{tabular}{lllll}
\hline Time $(\mathrm{min})$ & Mean & SD & $p$ value & Significant? \\
\hline $135^{\circ} \mathrm{C}$ & & & & \\
1 & 7.3 & 0.6 & 0.0002 & Yes \\
3 & 5.0 & 0.7 & 0.41 & No \\
5 & 4.9 & 0.4 & NA & No \\
$115{ }^{\circ} \mathrm{C}$ & & & & \\
1 & 7.8 & 0.8 & 0.0001 & Yes \\
3 & 6.6 & 0.3 & 0.0001 & Yes \\
5 & 5.2 & 0.4 & 0.207 & No \\
$95{ }^{\circ} \mathrm{C}$ & & & & \\
1 & 8.1 & 0.8 & 0.0001 & Yes \\
3 & 6.6 & 0.7 & 0.001 & Yes \\
5 & 5.8 & 0.5 & 0.01 & Yes \\
\hline
\end{tabular}


significantly different, apart from the samples produced at 3 min mixing at $135{ }^{\circ} \mathrm{C}$ or 5 min mixing at $115^{\circ} \mathrm{C}$. This means that according to the air voids criteria, AC containing 50\% RAP can be produced as low as $115{ }^{\circ} \mathrm{C}$ if it is mixed for $5 \mathrm{~min}$ without compromising the air voids, whereas other production conditions may significantly affect the air voids content.

\subsection{ITSM results}

The stiffness test results are presented in Fig. 8. A similar but inverse trend to the air voids can be seen, with an improvement in the ITSM as the temperature or mixing time increases. Furthermore, mixing at $135{ }^{\circ} \mathrm{C}$ for $5 \mathrm{~min}$ shows a comparable performance to the control HMA. These results give information on the blending process between the soft binder and the RAP binder. In the case of poor binder blending, the rejuvenator binder dominates the sample response since it represents the binder surrounding particle contacts, whereas much of the RAP binder is still acting like a black rock. However, as the mixing temperature, mixing time or both increases, the ITSM values increase, which means the two binders are blending and the hard binder is stiffening the soft binder during the mixing process. The binder stiffening process can be explained by the forced diffusion of the soft binder into the aged binder. The diffusion process is a function of mixing time, temperature, rejuvenator and aged binder properties [30]. Accordingly, the greater the mixing time and temperature, the higher the diffusion rate which ultimately leads to stiffening of the soft binder and increased ITSM. Moreover, it can be concluded from the results that when mixing at $95{ }^{\circ} \mathrm{C}$, relatively poor blending is achieved and the RAP is acting almost like a black rock, even after 5 min of mixing. Mixing at $115^{\circ} \mathrm{C}$ shows a partial participation of the RAP binder and the blending improves with mixing time. However, mixing at $135{ }^{\circ} \mathrm{C}$ for 3 or 5 min clearly indicates good blending between the two binders which is reflected in the ITSM results.

\subsubsection{Relations between ITSM, DOB and Pen}

Based on this evidence, stiffness measurements were used to quantify the DoB between RAP binder and the rejuvenator. The DoB is calculated as a percentage of the ITSM of the control mix using the following equation:

$\mathrm{DoB} \%=\mathrm{ITSM}_{\mathrm{AC}-50 \% \mathrm{RAP}} / \mathrm{ITSM}_{\text {Control }} \times 100 \%$

where $\mathrm{DoB} \%$ is the percentage of the degree of blending between RAP and soft binders,

Fig. 8 ITSM results

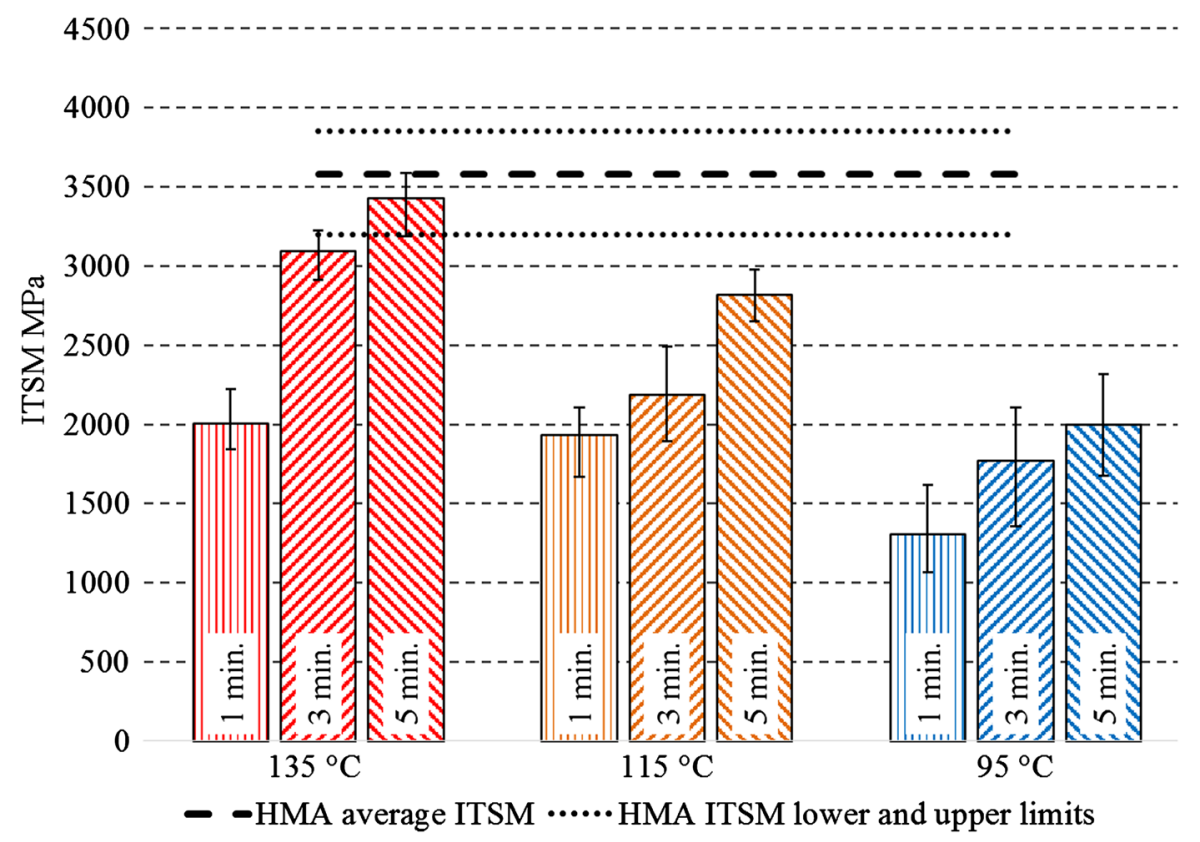


ITSM $_{\mathrm{AC}-50 \% \mathrm{RAP}}$ is the stiffness modulus of AC-RAP

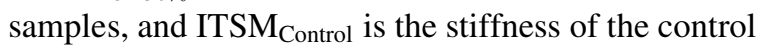
HMA. If the AC-RAP mix shows stiffness similar to the control mix, then the DoB is $100 \%$; lower AC-RAP stiffness means less blending. DoB results are presented in Fig. 9. It can be seen that $86-96 \%$ DoB was achieved when mixing for 3 or 5 min at $135{ }^{\circ} \mathrm{C}$, while $79 \%$ DoB was achieved when mixing at $115^{\circ} \mathrm{C}$ for 5 min. Other production conditions showed $60 \%$ or less DoB. However, these results were calculated based on the average ITSM results. To better analyse the DoB results, variability in ITSM results has to be considered. Figure 8 shows that all stiffness measurements of AC-RAP samples produced at $135^{\circ} \mathrm{C}$ when mixing for $5 \mathrm{~min}$ fall in the range of the control mix stiffness, suggesting no statistically significant difference between the two results. This means that these production conditions can be considered as optimised since they produce a mixture with statistically similar performance to the control HMA and the assumption of $100 \%$ RAP binder participation is therefore valid.

To further validate the results of this analysis, the relationship between ITSM at $20{ }^{\circ} \mathrm{C}$ and penetration grade was explored. Five binder grades between 33 and $188 \mathrm{dmm}$ penetration were used and AC samples fabricated with the same mix proportions and air voids content, and with virgin materials only. The ITSM values of these samples were then determined for at least three specimens per binder grade following the same procedure. The results are presented in Fig. 10. A power law equation was found to represent the relationship between binder grade and ITSM. Statistically speaking, $R^{2}$ equals 0.92 , which indicates a reasonable correlation. This relationship was then used to back-calculate and estimate the effective binder grade for the AC-RAP samples. Figure 11 shows the results of these back-calculated binder grades. It can be seen that the effective penetration of the samples produced at $135^{\circ} \mathrm{C}$ for 3 or $5 \mathrm{~min}$ of mixing reached that expected after full blending since the back-calculated binder grade is about $49 \mathrm{dmm}$, which matches the target binder grade. Furthermore, mixing at $115{ }^{\circ} \mathrm{C}$ for $5 \mathrm{~min}$ also came relatively close with $55.6 \mathrm{dmm}$. The results for back-calculated penetration grade provide a means of expressing the relative influence of rejuvenator and RAP binders, complementing the DoB values. The advantage of this approach is that it is possible to estimate binder grade and DoB without the need to extract and recover binder. Furthermore, the results presented in Fig. 11 show that the back-calculated binder grades for all samples were between 47 and $106 \mathrm{dmm}$, whereas the soft binder penetration was 188 , which means that even when mixing at $95^{\circ}$, part of the RAP binder is mobilized and participating in the mix.
Fig. 9 DoB calculation results

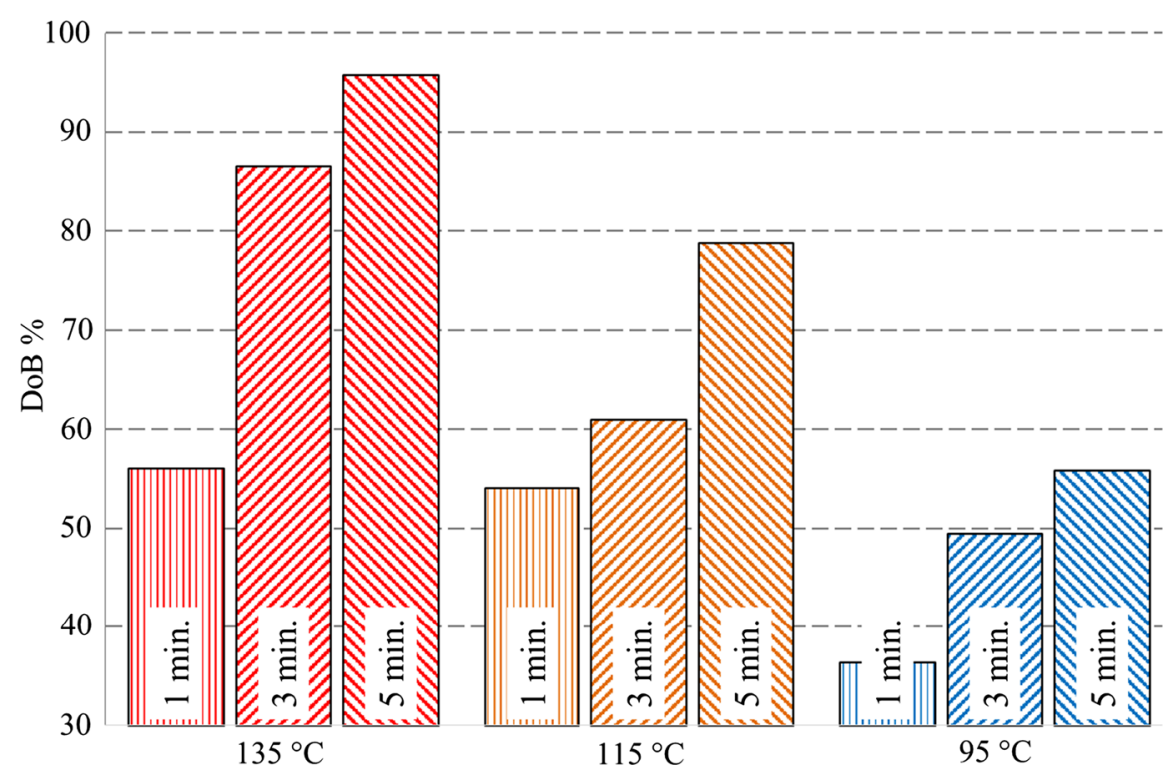


Fig. 10 Penetration and ITSM relationship

Fig. 11 Back-calculated penetration
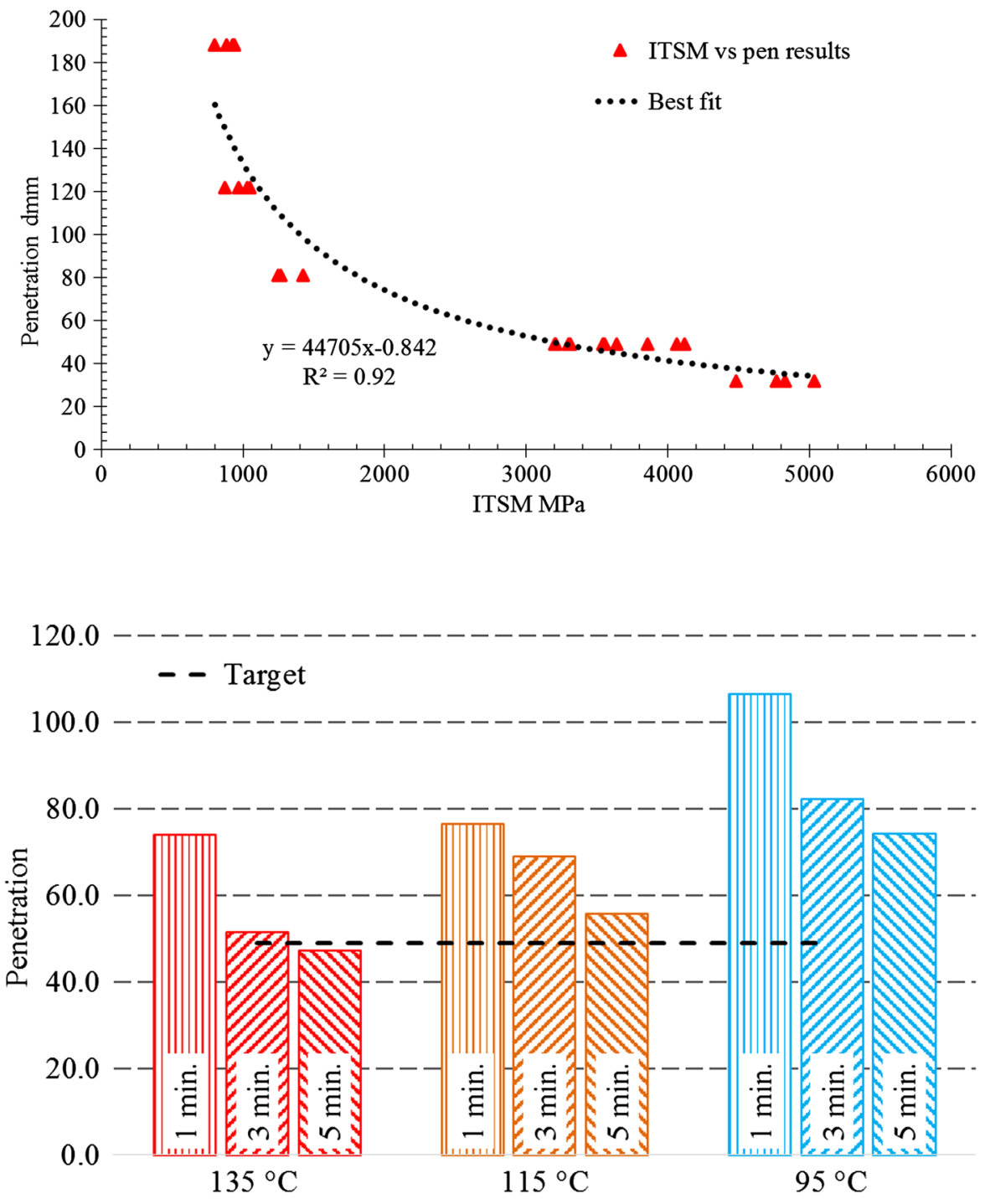

\subsection{ITS results}

The ITS results are presented in Fig. 12. The same trend can be identified; the higher the mixing time or temperature, the better the performance in terms of ITS. Mixing at $135{ }^{\circ} \mathrm{C}$ for $5 \mathrm{~min}$ achieved the target ITS, which was the strength of the control mix, whereas mixing at $135{ }^{\circ} \mathrm{C}$ for $3 \mathrm{~min}$ or at $115^{\circ} \mathrm{C}$ for 5 min resulted in $92 \%$ of the control ITS, approximately consistent with the ITSM results. However, as stated in the literature, the ITSM test is more sensitive to binder than the ITS, because the low strain in the ITSM test occurs mainly in the binder, with only slight aggregate movement during load application. For this reason, the ITS results were not used to estimate DoB or back-calculate the blended binder grade, but can be taken as an indicator of mixture performance to evaluate the fracture resistance of the AC-RAP mixtures.

\section{Conclusions}

A main reason that forces policy makers in limiting the amount of RAP content in surface courses is connected with uncertainties in the mix design such as 
Fig. 12 ITS results

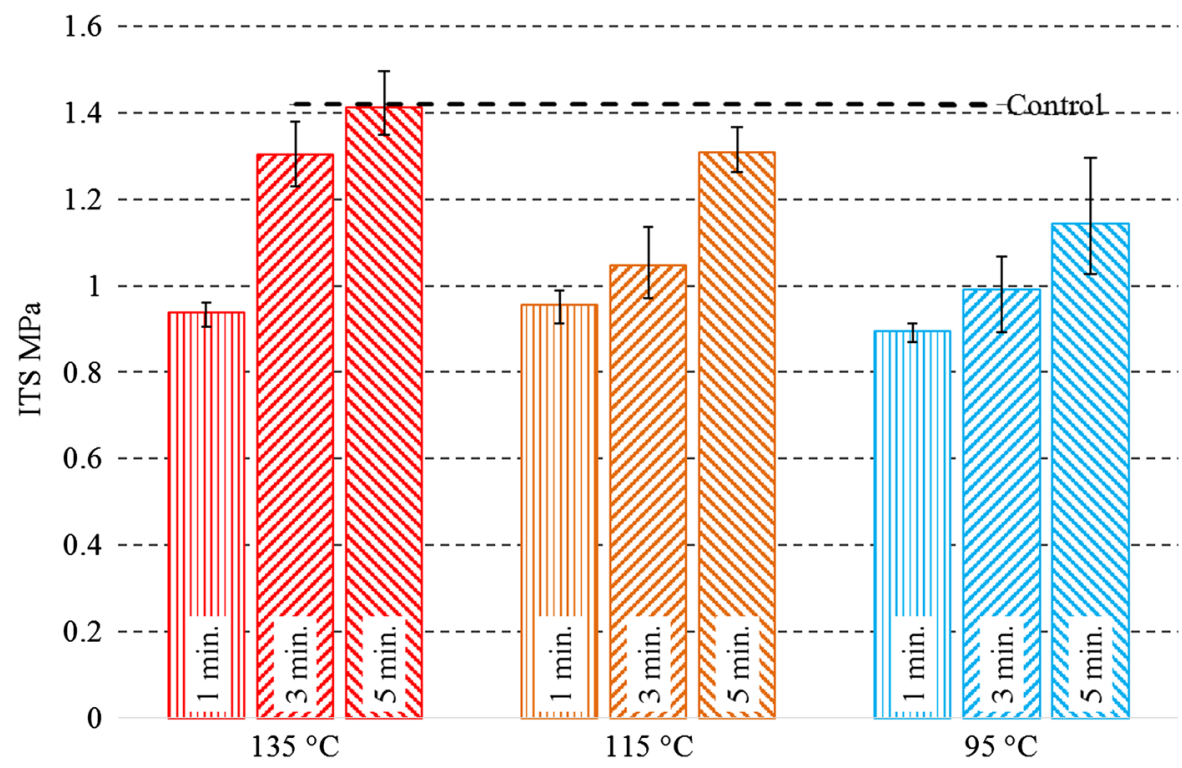

ideal mixing time and temperatures and the degree of mobilization of the RAP binder. This becomes even more complex when production temperatures are lowered within the warm mix region. This study shows that common assumptions, such as full blending, is rarely reasonable but could be practical if accurate design and processing conditions are used, even for asphalt mixes for surface courses incorporating high-content RAP and produced at WMA production temperatures. A 0/14 mm AC containing 50\% RAP was used in this study, and its laboratory performance evaluated based on ITSM and ITS indicators compared to a control mix with the same proportions but without RAP. The effect of two critical factors, mixing time and temperature, was studied and results provided a relation that allows mix designer to predict which conditions would make the hypothesis of full blending reasonable. This approach is based on ITSM or ITS results and allows evaluating the DoB and predict the effective penetration grade of the recycled mixture binder without the need to extract and recover any binder. Based on the results of this study, the main conclusions can be summarized are as follows:

1. Warm Asphaltic mixtures with high RAP content can be successfully produced with comparable stiffness and strength to traditional HMA if the production conditions are correctly designed.
2. The DoB was found to be a function of mixing time and temperature and it can be estimated from stiffness or strength measurements. The authors believe that ITSM measurements provide higher accuracy since this characteristic has been proved to be more sensitive to binder stiffness.

3. The hypothesis of full blending is rarely acceptable with the common mixing conditions guaranteeing asphalt plant productivity, however if these are optimised it can be plausible even within the warm mix region. For instance, in this study the ITSM results showed that full blending is a plausible assumption (DOB 96\%) for an AC with $50 \%$ RAP mixed at $135{ }^{\circ} \mathrm{C}$ only if 5 min were allowed for the mixing.

4. On the other hand, the black rock phenomenon is unlikely to happen in the warm region. In fact, results showed that even when mixing at temperatures as low as $95^{\circ} \mathrm{C}$ and for 1 min mixing time, the RAP binder was still partially mobilized with the DoB for this condition being 36\%. This is probably justified from the fact that at this temperature only the outer layer of RAP binder became active, and there was insufficient heat energy to activate the entire thickness. However, increasing mixing time improved this value due to the increased mechanical contact when mixing for an extended period. 
This investigation was tailored for one type of RAP and for one type of asphalt mixture only. Further study should look at extending this investigation looking at similar relations but covering a wider spectrum. Also considering that these findings might suggest that the full blending hypothesis is unpractical for the sake of asphalt plant productivity, it is suggested to investigate models and strategies aimed at improving this aspect on the basis of the presented results.

Acknowledgements This study is part of an ongoing Ph.D. research. The principal author would like to acknowledge the support of the Higher Committee for Education Development in Iraq for the Ph.D. scholarship.

\section{Compliance with ethical standards}

The authors declare that there is no issue concerning ethical standards.

Conflict of interest The authors declare that they have no conflict of interest.

Open Access This article is distributed under the terms of the Creative Commons Attribution 4.0 International License (http:// creativecommons.org/licenses/by/4.0/), which permits unrestricted use, distribution, and reproduction in any medium, provided you give appropriate credit to the original author(s) and the source, provide a link to the Creative Commons license, and indicate if changes were made.

\section{References}

1. EAPA (2010) The use of warm mix asphalt. European Asphalt Pavement Association, Brussels

2. Frank B et al (2011) Warm mix asphalt (WMA) emission reductions and energy savings. Auburn University, Auburn

3. Stotko O (2011) Energy and related carbon emissions reduction technologies for hot mix asphalt plants. In: 10th conference on asphalt pavements for Southern AfricaCAPSA, Winterton, South Africa

4. Hansen KR, Copeland A (2015) Asphalt pavement industry survey on recycled materials and warm-mix asphalt usage5th annual survey. National Asphalt Pavement Association, Washington, DC, USA

5. Copeland A (2011) Reclaimed asphalt pavement in asphalt mixtures: state of the practice. Federal Highway Administration, Washington

6. Kandhal PS, Mallick RB (1997) Pavement recycling guidelines for state and local governments. National Center for Asphalt Technology, Washington

7. Giani MI et al (2015) Comparative life cycle assessment of asphalt pavements using reclaimed asphalt, warm mix technology and cold in-place recycling. Resour Conserv Recycl 104:224-238
8. Al-Qadi IL, Elseifi M, Carpenter SH (2007) Reclaimed asphalt pavement-a literature review. Illinois Center for Transportation, Rantoul

9. Lo Presti D et al (2016) Towards $100 \%$ recycling of reclaimed asphalt in road surface courses: binder design methodology and case studies. J Clean Prod 131:43-51

10. Shirodkar $P$ et al (2011) A study to determine the degree of partial blending of reclaimed asphalt pavement (RAP) binder for high RAP hot mix asphalt. Constr Build Mater 25(1):150-155

11. Coffey S et al (2013) Determining the impact of degree of blending and quality of reclaimed asphalt pavement on predicted pavement performance using pavement $\mathrm{ME}$ design. Constr Build Mater 48:473-478

12. Bowers BF, Moore J, Huang B, Shu X (2014) Blending efficiency of reclaimed asphalt pavement: an approach utilizing rheological properties and molecular weight distributions. Fuel. https://doi.org/10.1016/j.fuel.2014.05.059

13. Bressi S, Dumont A-G, Pittet M (2015) Cluster phenomenon and partial differential aging in RAP mixtures. Constr Build Mater 99:288-297

14. Hassan NA et al (2015) Effect of mixing time on reclaimed asphalt mixtures: an investigation by means of imaging techniques. Constr Build Mater 99:54-61

15. British Standards Institution, BS EN 13108-1:2006 (2006) Bituminous mixtures-material specifications-part one: asphalt concrete. BSI, United Kingdom

16. British Standards Institution, BS 4987-1:2005 (2005) Coated macadam (asphalt concrete) for roads and other paved areas_-part 1: specification for constituent materials and for mixtures. BSI, United Kingdom

17. British Standards Institution, BS EN 12697-5-2009 (2009) Bituminous mixtures- test methods for hot mix asphaltpart 5: determination of the maximum density. BSI, United Kingdom

18. Yildirim Y, Solaimanian M, Kennedy TW (2000) Mixing and compaction temperatures for hot mix asphalt concrete. Center for Transportation Research, University of Texas at Austin

19. British Standards Institute, BS EN 12697-3-2013 (2013) Bituminous mixtures-test methods for hot mix asphaltpart 3: bitumen recovery: rotary evaporator. BSI, United Kingdom

20. British Standards Institute, BS EN 12697-1:2012 (2012) Bituminous mixtures- test methods for hot mix asphaltpart 1: soluble binder content. BSI, United Kingdom

21. British Standards Institute, BS EN 12697-2-2015 (2015) Bituminous mixtures-test methods-part 2: determination of particle size distribution. BSI, United Kingdom

22. Hossain M, Maag R, Fager G (2010) Handbook of superpave volumetric asphalt mixture design and analysis. Superpave Certification Training Manual, Kansas State University, Manhattan, Kansas

23. AASHTO (2013) Standard specification for superpave volumetric mix design, M 323. American Association of State Highway and Transportation Officials (AASHTO), USA

24. British Standards Institution, BS EN 12697-6-2012 (2012) Bituminous mixtures-test methods for hot mix asphaltpart 6: determination of bulk density of bituminous specimens. BSI, United Kingdom 
25. Thom N (2014) Principles of pavement engineering, 2nd edn. ICE Publishing, London

26. British Standards Institution, BS EN 12697-26:2004 (2004) Bituminous mixtures - test methods for hot mix asphaltpart 26: stiffness. BSI, United Kingdom

27. Khosla NP, Harikrishnan KI (2007) Tensile strength-a design and evaluation tool for superpave mixtures. Department of Civil Engineering, North Carolina State University
28. British Standards Institution, BS EN 12697-23-2003 (2003) Bituminous mixtures-test methods for hot mix asphaltpart 23: determination of the indirect tensile strength of bituminous specimens. BSI, United Kingdom

29. Bressi S et al (2015) Particle clustering phenomena in hot asphalt mixtures with high content of reclaimed asphalt pavements. Constr Build Mater 100:207-217

30. Zaumanis M, Mallick RB (2013) Finite element modeling of rejuvenator diffusion in RAP binder film—simulation of plant mixing process. Springer, Dordrecht 